ARTICLE

\title{
Fibroblast fusion to the muscle fiber regulates myotendinous junction formation
}

\author{
Wesal Yaseen ${ }^{1}$, Ortal Kraft-Sheleg ${ }^{1}$, Shelly Zaffryar-Eilot', Shay Melamed', Chengyi Sun (1) ${ }^{2}$, \\ Douglas P. Millay (i) ${ }^{2,3} \&$ Peleg Hasson ${ }^{1 凶}$
}

\begin{abstract}
Vertebrate muscles and tendons are derived from distinct embryonic origins yet they must interact in order to facilitate muscle contraction and body movements. How robust muscle tendon junctions (MTJs) form to be able to withstand contraction forces is still not understood. Using techniques at a single cell resolution we reexamine the classical view of distinct identities for the tissues composing the musculoskeletal system. We identify fibroblasts that have switched on a myogenic program and demonstrate these dual identity cells fuse into the developing muscle fibers along the MTJs facilitating the introduction of fibroblast-specific transcripts into the elongating myofibers. We suggest this mechanism resulting in a hybrid muscle fiber, primarily along the fiber tips, enables a smooth transition from muscle fiber characteristics towards tendon features essential for forming robust MTJs. We propose that dual characteristics of junctional cells could be a common mechanism for generating stable interactions between tissues throughout the musculoskeletal system.
\end{abstract}

\footnotetext{
${ }^{1}$ Department of Genetics and Developmental Biology, The Rappaport Faculty of Medicine and Research Institute, Technion - Israel Institute of Technology, Haifa, Israel. ${ }^{2}$ Division of Molecular Cardiovascular Biology, Cincinnati Children's Hospital Medical Center, Cincinnati, OH, USA. ${ }^{3}$ Department of Pediatrics,

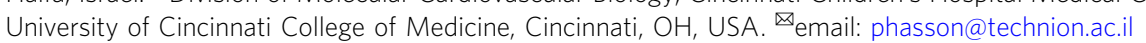


T ransplantation experiments in avian embryos as well as genetic investigations in mice performed over the last few decades identified the lineages, that make up the vertebrate limb musculoskeletal system. These have demonstrated that while myogenic precursor cells are somite-derived, the connective tissues, tendons, and bones are derived from the lateral plate mesoderm $(\mathrm{LPM})^{1-4}$. However, single cell resolution techniques allow us to revisit these early observations and focus on specific regions, such as the sites of interaction between the distinct tissues to resolve their cellular contributions. One such focal point is the myotendinous junctions (MTJs).

Although being critical for muscle functioning in transmitting the force generated by the muscle to the tendons and skeletal elements, our understanding of the mechanisms that underlie MTJ development and maintenance are still relatively unclear. Importantly, while myofiber tips along the MTJs serve as the sites of interaction with the tendons, during embryonic and neonatal development when myofibers elongate via myoblasts' fusion, the majority of fusion events also take place at these regions ${ }^{5-9}$. Accordingly multiple signaling pathways such as BMP and FGF signaling are tightly coordinated along the fiber tips ${ }^{10,11}$ although their exact contribution to fusion, myofiber elongation, and/or MTJ formation and maintenance is still unclear.

In this work, we examine the mechanisms underlying MTJ formation. We carry out a single cell transcriptome (scRNAseq) analysis of the MTJ region. This analysis reveals the presence of a unique cluster of cells expressing both myogenic as well as fibroblastic characteristics. Interestingly, cells in this cluster also express multiple genes known to be associated with MTJs. Surprisingly, this analysis further identifies that the secreted extracellular matrix (ECM) modifying enzyme Lysyl oxidase-Like 3 (LoxL3), an essential enzyme required for MTJ formation, that is secreted from myofiber tips ${ }^{12}$ is transcribed by fibroblasts and not by myogenic cells. Using fluorescent in situ hybridization (FISH) analyses and tracing of fibroblast nuclei in vitro and in vivo within the developing muscle, we identify a mechanism of horizontal RNA transfer by which LPM-derived fibroblasts transdifferentiate, switch on myogenic characteristics and fuse into the myofibers along the MTJs. We suggest recruitment of LPMderived cells from muscle boundaries and their fusion into the myofibers is essential for normal MTJ development ensuring proper localization of proteins along these junctions.

\section{Results}

scRNAseq reveals cells with dual identities. To investigate the cellular contributions to the MTJs, we carried out a single cell transcriptome analysis of the muscle-tendon region at P0 (https:// www.ncbi.nlm.nih.gov/geo/query/acc.cgi?acc=GSE168153). At this stage, MTJs have already formed yet myoblast fusion at this region is high. Single cells (i.e., without contribution of syncytial myofibers) were used for the analysis. Transcriptome analysis identified 21 independent clusters (Fig. 1a). Clusters expressing the pan muscle connective tissue interstitial marker PDGFR $\alpha$ (marked as fibroblasts in the UMAP; Fig. 1a) and Osr1 were identified. These included muscle connective tissue fibroblasts with high $T c f 4$ (Tcf7l2) or fibro-adipogenic precursors (FAPs) expressing ScaI (Ly6a). These fibrogenic clusters expressed significantly distinct transcriptomes from those enriched for myogenic markers (consisting of myoblasts progenitors and Myogenin expressing myocytes; Supp. Fig. 1a). We could further identify tenocytes, endothelial, and mural cells (Supp. Fig. 1b-d). A small number of cells co-expressing myogenic and fibrogenic markers were identified in multiple clusters. Notably, while generally the above clusters could be associated with a single identity, a small number of cells clustered together ( $n=38$; dual identity cells) expressing both fibrogenic identity markers as PDGFR $\alpha$, Osr1, Colla1, and Dcn but also myogenic markers such as Pax7, Myf5, MyoD1, M-Cadherin (Cdh15) and others (Fig. 1b-e and Supp. Fig. 1a-g). Interestingly, cells in this cluster also express multiple MTJ and tenogenic markers such as Ankrd1, Thbs4, Bgn, and Tn-C, respectively ${ }^{13-16}$. Notably, some of these markers are specific to this cluster suggesting these dual identity cells form an independent cell cluster (Fig. 1e).

To further characterize the dual identity cells, we used Ingenuity Pathway Analysis (IPA) software. When comparing transcriptomes of this cluster to that of the myogenic clusters using the upstream regulator analysis tool, we find TGF $\beta$, previously associated with MTJ formation ${ }^{17}$, being the most highly upregulated pathway in this cluster $(Z$-score $5.528, p$ value $\left.1 \times 10^{-37}\right)$. This analysis further suggests BMP signaling, known to be highly activated along myofiber tips ${ }^{11}$ (and Esteves de Lima, co-submitted), is activated in the dual identity cells ( $Z$-score $3.544, p$ value $\left.5.7 \times 10^{-7}\right)$. TGF $\beta$ and BMP signaling were also suggested to be activated when comparing these unique progenitors to fibrogenic clusters although to a lower extent ( $Z$-score $1.69, p$ value $3 \times 10^{-10} ; Z$-score $2.13, p$ value $2.95 \times 10^{-3}$, respectively), but not to the myogenic clusters. Altogether these results suggest these two cascades are significantly activated in the cluster containing the dual identity cells.

The dual identity cells expressed genes associated with myogenic proliferation (e.g., Pax7 and Myf5) and early differentiation (e.g., MyoD1 but not Myogenin). Accordingly, IPA analysis suggests they are much more motile and proliferative. WEB-based Gene Set Analysis Toolkit (WebGestalt ${ }^{18}$ ) using the differentially expressed genes (DEGs) in this cluster versus those of the myogenic or fibrogenic clusters was further carried out. Changes in ECM composition of the dual identity clustered cells only vs. myogenic clusters were highlighted. These differences further demonstrate that cells in this unique cluster express high levels of ECM genes more similar to that observed in fibrogenic and tendon cells reinforcing the notion these cells form a transition between the myofiber and the tendon within the MTJ.

Remarkably, RNA velocity, an analysis which predicts future cell fate based on abundance of nascent (unspliced) and mature (spliced) mRNA forms in single cell data ${ }^{19}$ suggests that the progenitors with a dual identity stem from fibrogenic clusters moving towards the myogenic identity (Fig. If and Supp. Fig. $1 \mathrm{~h}-\mathrm{i}$ ). Hence raising the possibility that these progenitors transdifferentiate from fibroblasts into myogenic progenitors.

LoxL3 RNA is expressed by muscle interstitial cells. To begin to test the transdifferentiation idea, we probed a system where we previously demonstrated that the ECM modifying enzyme LoxL3 plays an essential role in MTJ development. In LoxL3 mutant embryos, myofibers do not properly anchor along the MTJs ${ }^{12}$ (Supp. Fig. 2a-c). The scRNAseq analysis revealed LoxL3 transcripts are primarily expressed in fibrogenic clusters and not in the myogenic ones (Supp. Fig. 1j). Surprisingly, this expression pattern contrasts with the expression of LoxL3 protein, which is expressed inside the myofiber tips (Supp. Fig. 2d-k) and being a secreted ECMmodifying enzyme is presumably secreted from their tips to modify the MTJ matrix promoting myofiber anchorage along the MTJ.

To verify the scRNAseq results that suggest LoxL3 RNA is expressed primarily by fibroblasts we carried out FISH analysis on tissue sections. In accordance with the above RNA sequencing results and in contrast to LoxL3 protein expression, we find LoxL3 RNA is mostly expressed adjacent to the muscle tips yet primarily outside of the myofibers (Fig. 2a, b).

This unexpected observation of different protein and RNA expression lead us to further explore LoxL3 RNA localization in a more controlled environment. We took advantage of our previous 
a.

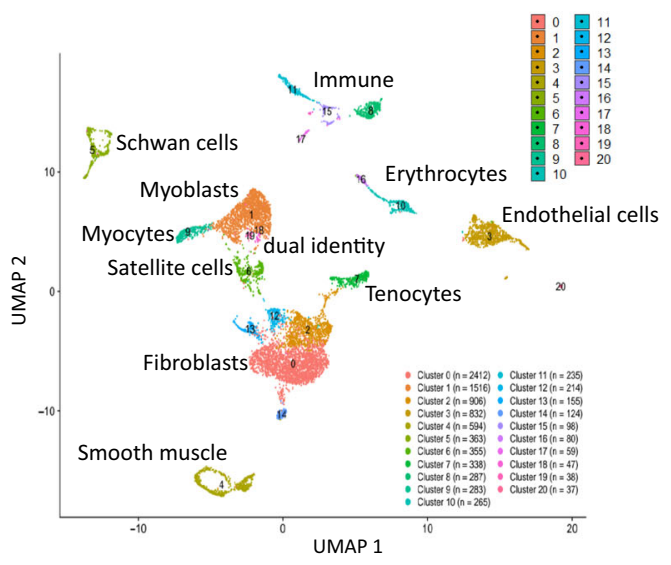

b.

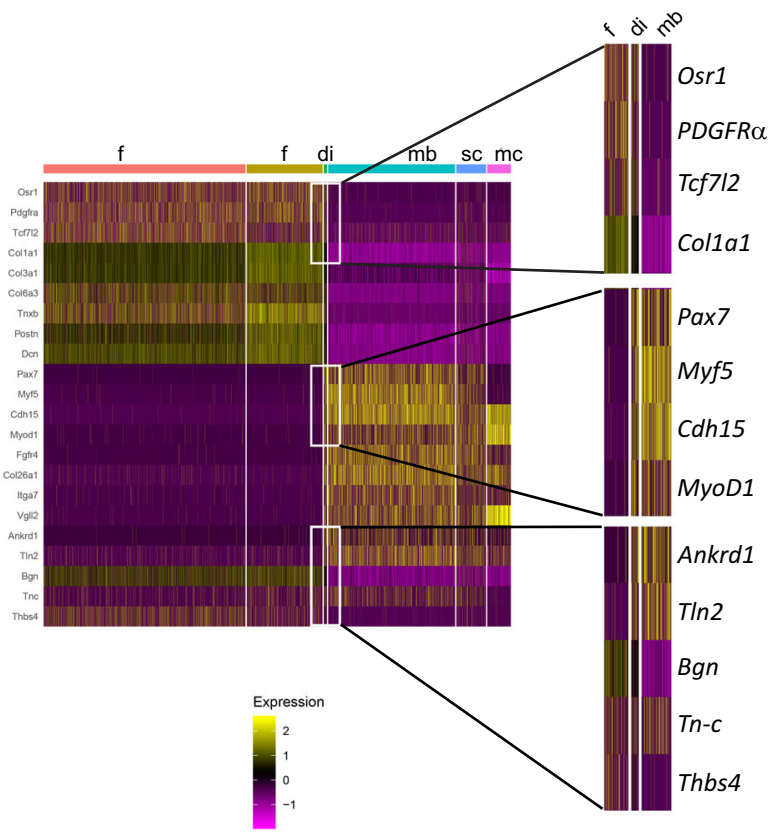

c.

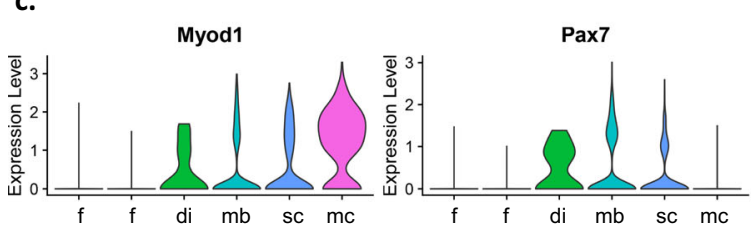

d.

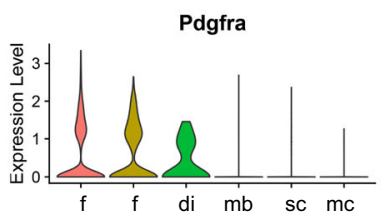

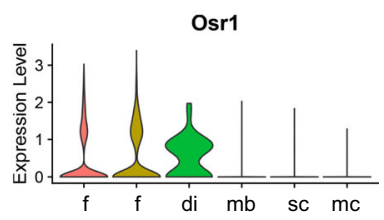

e.
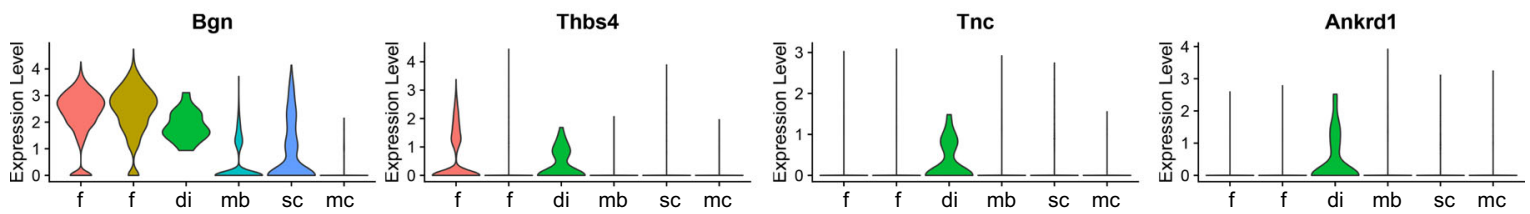

f.

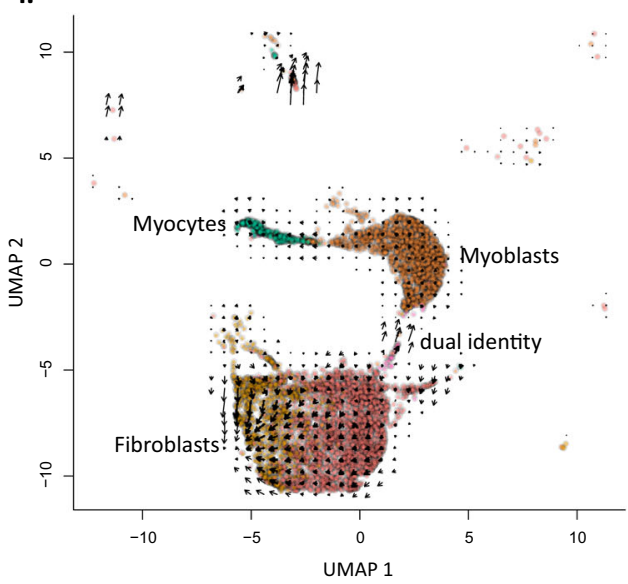

Fig. 1 scRNA sequencing reveals cells with dual myogenic and interstitial transcriptional identities. UMAP depicting cell clusters (a) heat map (b) and violin plots of distinct genes associated with myogenesis (c) FAPs/fibroblasts (d), and tendon and MTJs (e). UMAP of RNA velocity analysis of myogenic $(1,6,9)$, fibrogenic $(0,2)$ and dual identity clusters (f). f fibroblasts, mb myoblasts, mc myocytes, sc satellite cells, di dual identity. 

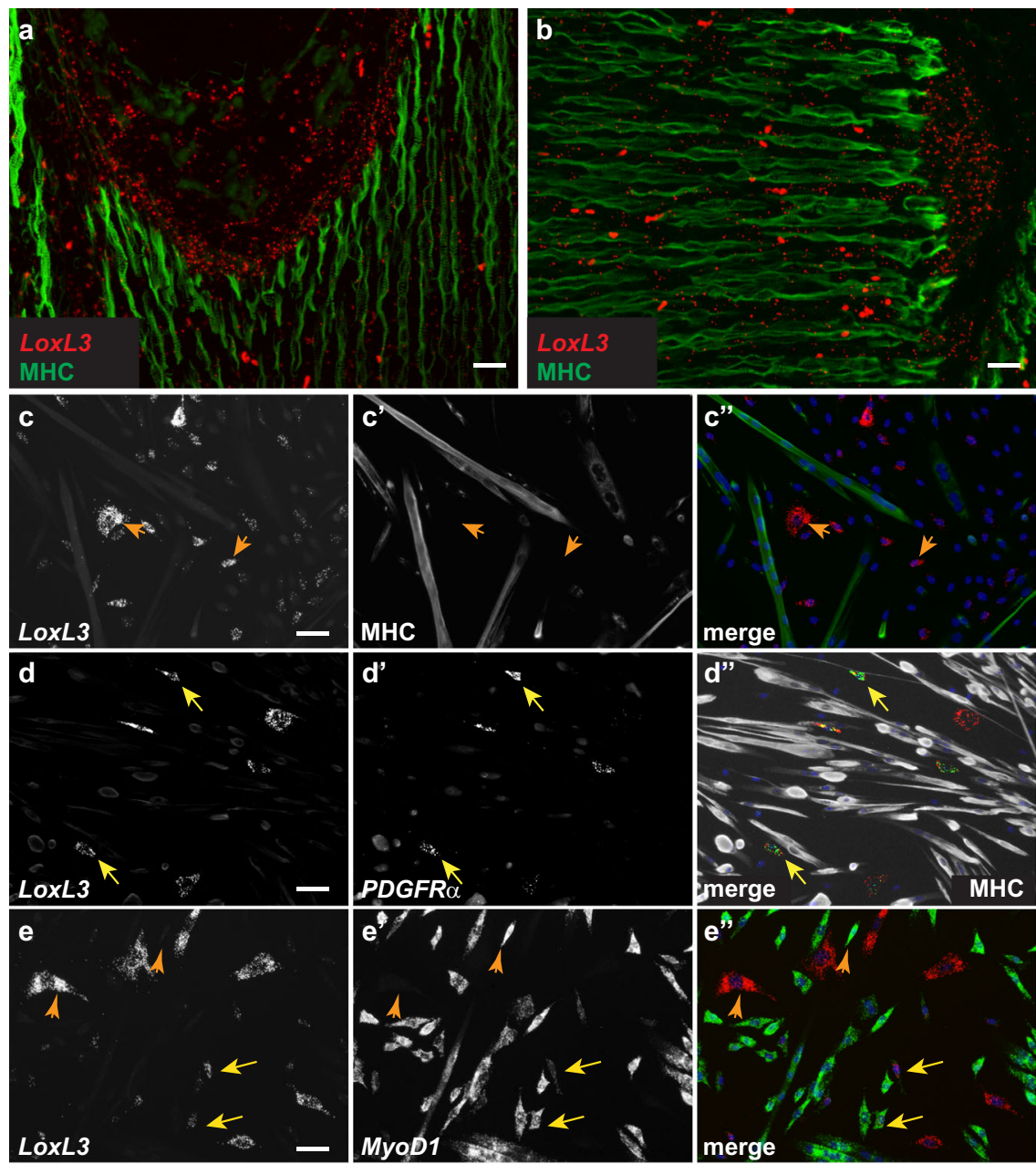

Fig. 2 LoxL3 RNA is expressed in muscle interstitial cells. FISH staining for LoxL3 RNA (red) and MHC immunostaining (green) at E15.5 demonstrates LoxL3 is expressed close to myofiber tips yet primarily outside of the myofibers (a, b). FISH for LoxL3 (c) or also for PDGFR $(\mathbf{d})$ and anti-MHC staining demonstrates that LoxL3 RNA is not expressed in myofibers but in PDGFR $\alpha$ expressing interstitial cells. FISH for LoxL3 and MyoD1 (e) reveals that most cells express either one of these markers (orange arrows), some express both (yellow arrows). Images shown are representative of at least three independent embryos or cell isolation experiments. Scale bars $=50 \mu \mathrm{m}$.

observations demonstrating that in in vitro differentiated myotubes, LoxL3 protein localization is maintained at the tips ${ }^{12}$. We therefore monitored LoxL3 RNA in primary cultures containing myoblasts, myotubes (marked by myosin heavy chain, MHC) and interstitial fibroblasts/FAPs (marked by PDGFR $\alpha$, Supp. Fig. 2l) (Fig. 2c-e). In accordance with the in vivo results, no LoxL3 RNA expression was observed in myotubes (Fig. 2c- $\mathrm{c}^{\prime \prime}$ ). In contrast, LoxL3 mRNA was primarily observed in PDGFR $\alpha$ expressing fibroblasts/FAPs interstitial cells (Fig. 2d-d"). Staining for LoxL3 and MyoD1 RNAs revealed that most cells expressed either marker-myogenic cells expressed $M y o D 1$ whereas fibroblasts/FAPs expressed LoxL3 (Fig. 2e-e" ; orange arrowheads). Notably, $\sim 33 \%$ of the LoxL3 expressing cells also expressed MyoD1 (Fig. 2e-e" ; yellow arrows). Altogether these results demonstrate that in contrast to its protein expression, LoxL3 RNA is primarily expressed by bona fide MCT fibroblasts/FAPs some of which also express MyoD1.

LPM-derived fibroblasts fuse to growing myofibers. The observation that LoxL3 and PDGFR $\alpha$ expressing cells could also express myogenic markers (Fig. 2e), together with the results of the RNA velocity analysis (Fig. 1f), led us to test whether these cells are LPM-derived fibroblasts that have switched on a myogenic program. Towards testing this hypothesis, we took advantage of the $\operatorname{Pr} x 1^{\text {Cre } 20}$, a LPM-specific Cre driver line driven by a Prrx 1 limb enhancer ${ }^{21}$ previously suggested not to be expressed in myogenic cells ${ }^{20,22,23}$. scRNAseq confirmed Prrx1 is expressed by fibrogenic and not myogenic cells. Notably this analysis further demonstrated Prrxl is also expressed by the dual identity cells (Supp. Fig. 3a) further reinforcing the above observations (Fig. 1f) that these cells are derived from the LPM.

To further test the validity of this Cre line in vivo, we crossed it to the Rosa $a^{n T-n G}$ reporter line ${ }^{24}$. In this reporter, all nuclei express tdTomato however upon Cre activity nuclear EGFP is switched on instead. Hence it enables the identification of LPM-derived nuclei in syncytial myofibers. Section immunostaining for Laminin marking the myofibers of E18.5 embryonic limb muscles demonstrates that no LPM-derived nuclei (EGFP-expressing) are found within the fibers away from the MTJ (Supp. Fig. 3b, $b^{\prime}$ ). Along these lines, immunostaining for Pax3 marking myogenic precursors at E10.0 show no overlap with the LPM-derived EGFP expressing cells (Supp. Fig. 3c).

Our results above suggest that LPM-derived cells can transdifferntiate and switch on a myogenic program. To test whether this takes place, we crossed the Prxicre to the Rosa26R tdTomato 25 , a cytoplasmic reporter, resulting in tdTomato expressing LPM-derived cells. FISH analysis was carried out on cultured cells derived from limb muscles. We find that MyoD1 

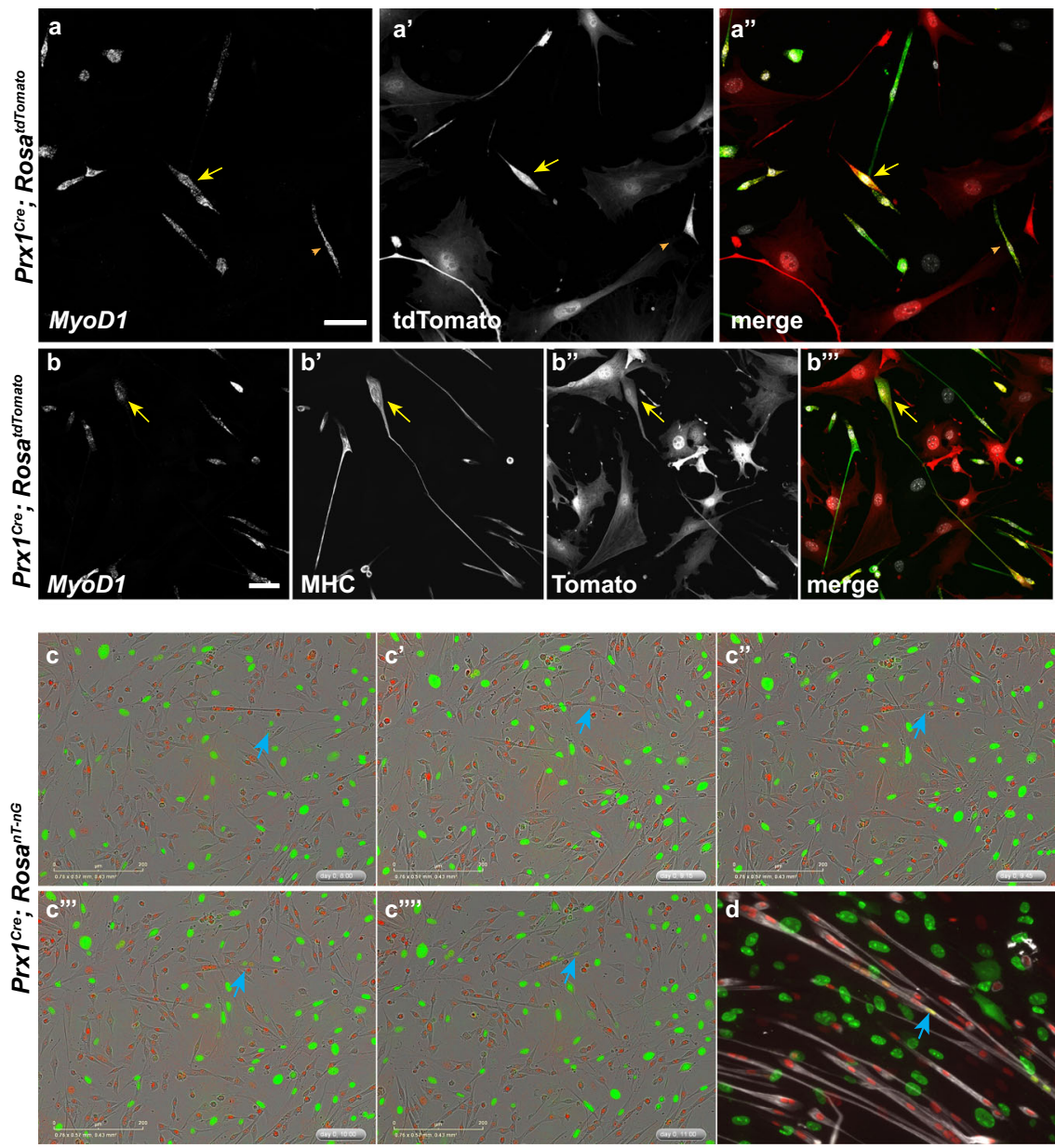

Fig. 3 Cultured muscle interstitial cells fuse into growing myotubes. Primary culture of myoblasts and interstitial cells derived from Prx 7 Cre; RosatdTomato following $24 \mathrm{~h}$ of differentiation demonstrates the presence of tdTomato expressing MyoD1 (yellow) and MHC (green) expressing myofibers (a, b). Snapshots of live-cell imaging of $\operatorname{Prx}_{7}{ }^{C r e}$; Rosat-ng-derived primary culture after $48 \mathrm{~h}$ in differentiation media revealing fusion of fibroblast (blue arrow) into

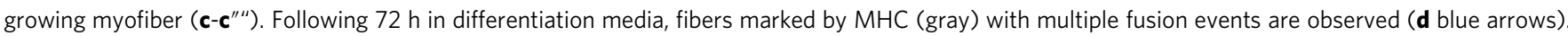
Shown are representative images of FISH and immunostaining results of at least three independent cell isolation experiments from the distinct genotypes. Scale bar in $\mathbf{a}, \mathbf{b}=50 \mu \mathrm{m}$ and in $\mathbf{c}=200 \mu \mathrm{m}$.

RNA is expressed also in a subset of LPM-derived fibroblasts (Fig. 3a- $\mathrm{a}^{\prime \prime}$ ), and that tdTomato MHC expressing myotubes do form in these cultured cells (Fig. 3b-b'"').

The observation of tdTomato-expressing myotubes could be a consequence of several mechanisms that facilitate the introduction or transfer of the fibroblast-derived tdTomato RNA into the myofibers. Such mechanisms could include fusion of fibroblasts into the myofibers, extracellular vesicles released to the media that are then taken up by the myofibers or cellular extensions such as cytonemes between cell types ${ }^{26-28}$. The observation of adjacent $M y o D 1$ expressing cells, where one is tdTomato-positive and the other negative (Fig. 3a; yellow arrow) suggested this is not a random event and may result from a regulated process such as cell fusion, an event that is essential for myofiber growth. To directly test whether fibroblasts fuse into growing fibers, we crossed the Rosant-ng reporter line that enables tracking of the distinct nuclei even in syncytia such as myofibers. Single cells were isolated from limb muscles of $\mathrm{Prxl}_{1}{ }^{\mathrm{Cre}} ; \mathrm{Ros}^{\text {nt-ng }} \mathrm{P} 0$ neonates and cultured in low serum to facilitate myogenic differentiation. In these cultures, all myogenic cells' nuclei are tdTomatoexpressing while all LPM-derived fibroblasts/FAPs' nuclei express EGFP. Culturing of sorted tdTomato-only or EGFP-only cells (Supp. Fig. 3d) demonstrates that, as expected, late or random Cre activity (and hence loss of tdTomato and gain of EGFP) does not occur in these culture conditions (Supp. Fig. 3e-g"). Live cell imaging confirms EGFP expressing fibroblasts/FAPs fuse into growing myofibers (Fig. 3c- $\mathrm{c}^{\prime \prime \prime}$; blue arrow and Supp. Movie1). Following $72 \mathrm{~h}$ of in vitro differentiation $\sim 7 \%$ of MHC expressing myotubes $(n=276)$ harbor at least one EGFP expressing nucleus derived from such a fusion event (Fig. 3d).

That LPM-derived interstitial cells can fuse into growing myotubes raised the possibility that fibroblast-specific RNAs would be transferred along, thus facilitating the expression of fibroblast/FAP-associated genes such as LoxL3 and PDGFR $\alpha$ within the myotube. Accordingly, we find that only in tdTomatopositive fibers (i.e., have a contribution of at least one fibroblast) the fibroblast/FAP-specific RNAs of LoxL3 and PDGFR $\alpha$ are found (Fig. 4a, b and Supp. Fig. 4). To test whether the LPMderived cells that have fused into the myotubes instruct other nuclei to transcribe the fibroblast/FAP-specific RNAs or whether they are the ones that actively transcribe it, we monitored the expression of PDGFR $\alpha$ in myotubes derived from $\mathrm{Prxl}{ }^{\mathrm{Cre}}$; Rosa ${ }^{\text {nt- }}$ $n g$ mice. We find that active transcription as monitored by two fluorescent foci inside the nucleus, occurs only in the EGFPpositive nuclei (Fig. $4 \mathrm{c}, \mathrm{d}^{\prime \prime}$ ). Overall, these results reveal that not only do LPM-derived interstitial cells fuse into growing fibers but that they also maintain, at least to some extent, their initial identities' transcriptional program. 

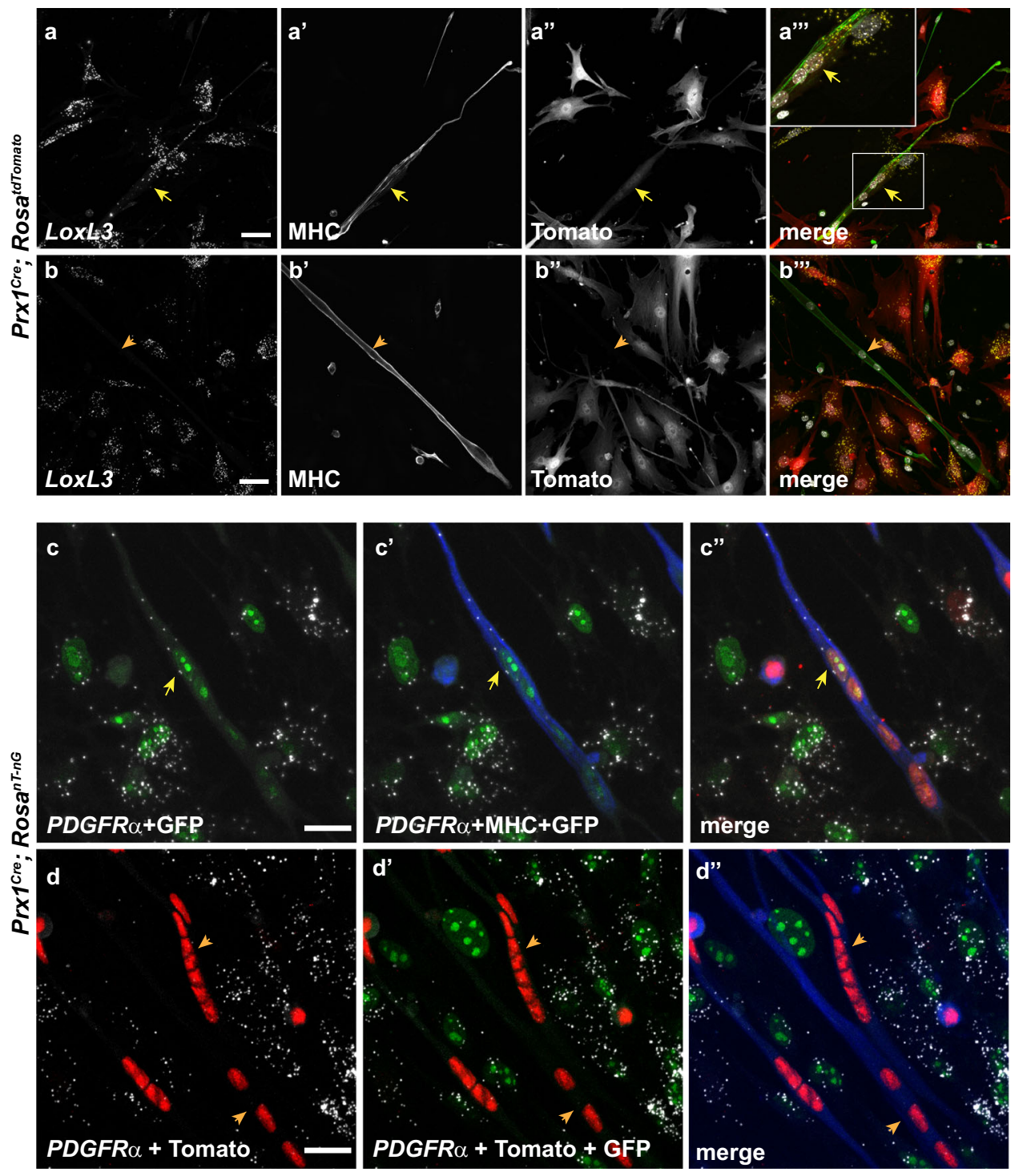

Fig. 4 Fused LPM-derived cells actively transcribe and contribute fibroblast-associated genes to growing myotubes. FISH for $L O x L 3$ (a-b"') or PDGFR $\alpha$ $\left(\mathbf{c}-\mathbf{d}^{\prime \prime}\right)$ on primary cultured myoblasts and interstitial cells derived from Prx 7 Cre; RosatdTomato (a, b) or Prx 7 Cre; Rosant-ng (c, d) following $48 \mathrm{~h}$ of differentiation demonstrates that only in fibers where LPM-derived cells have fused (yellow arrows) but not in tdTomato-negative (b-b'") or GFP-negative (d-d"d ) are LoxL3 (a) or PDGFR $(\mathbf{c})$ RNA are observed. MHC in gray or green $(\mathbf{a}, \mathbf{b})$ or blue $(\mathbf{c}, \mathbf{d})$. Shown are representative images of FISH and immunostaining results of at least three independent cell isolation experiments from the distinct genotypes. Scale bar $=50 \mu \mathrm{m}$.

A series of experiments marking proliferating cells, have demonstrated that within the elongating muscles, progenitors fuse to elongating myofibers primarily along the tips adjacent to the MTJs $\mathrm{s}^{5-7}$. Although the previous experiments did not monitor the identity of these progenitors, Prxi Cre; Rosant-ng enable dissection of whether some of the fused cells are LPM-derived fibroblasts. Analysis of $\mathrm{Prxl}_{1}^{\mathrm{Cre}}$; Rosa ${ }^{\text {nt-ng }}$ mice demonstrate that, as expected, the myofibers' nuclei are primarily tdTomatopositive while EGFP expressing nuclei are found along the MTJs and in between myofibers (Fig. 5a). Should fibroblasts fuse into the growing myofibers also in vivo, we would expect that in proximity to the myofiber tips, adjacent to the MTJs, EGFPexpressing nuclei will be found. IMARIS 3D analysis of confocal images derived from wholemount immunostaining of muscles, demonstrates this is the case and the EGFP nuclei are primarily located close to the MTJs (Fig. 5a, b and Supp. Fig. 5a-a' and Supp. Movies 2-3). Late activation of $\mathrm{Prxl}^{\mathrm{Cre}}$ also in myogenic cells could, in principle, lead to EGFP expressing nuclei within the myofibers. However, should this be the case, then we would expect these EGFP nuclei to be present throughout the myofibers. Our cell culture analyses (Supp. Fig. $3 \mathrm{e}-\mathrm{g}^{\prime \prime}$ ) as well as our in vivo stainings which show that even in late stages of embryogenesis EGFP expressing nuclei are not present throughout the myofibers (Supp. Fig. 3) but only along the MTJs, suggests such late activation does not occur. Altogether these results demonstrate that fibroblast fusion into myofibers occurs as part of the normal developmental program, and confirm in vivo the in vitro observations of the dual origin of cells that make up the myofiber.

Fusion between myoblasts has been shown to be dependent on the transmembrane fusogene myomaker $(\text { Mymk })^{29}$. To test whether the LPM-derived cells fuse into the myofiber in a Mymk-dependent manner we deleted Mymk in the LPM lineage using the $\operatorname{Pr} x_{1} \mathrm{Cre}$, however no MTJ abnormalities were observed (Supp. Fig. 5b, c). Overall, these observations suggest that the fusion of LPM-derived cells into the myofiber is dependent on a mechanism not involving Mymk in fibroblasts and potentially consistent with the lack of need for Mymk in myofibers for fusion ${ }^{30,31}$. 

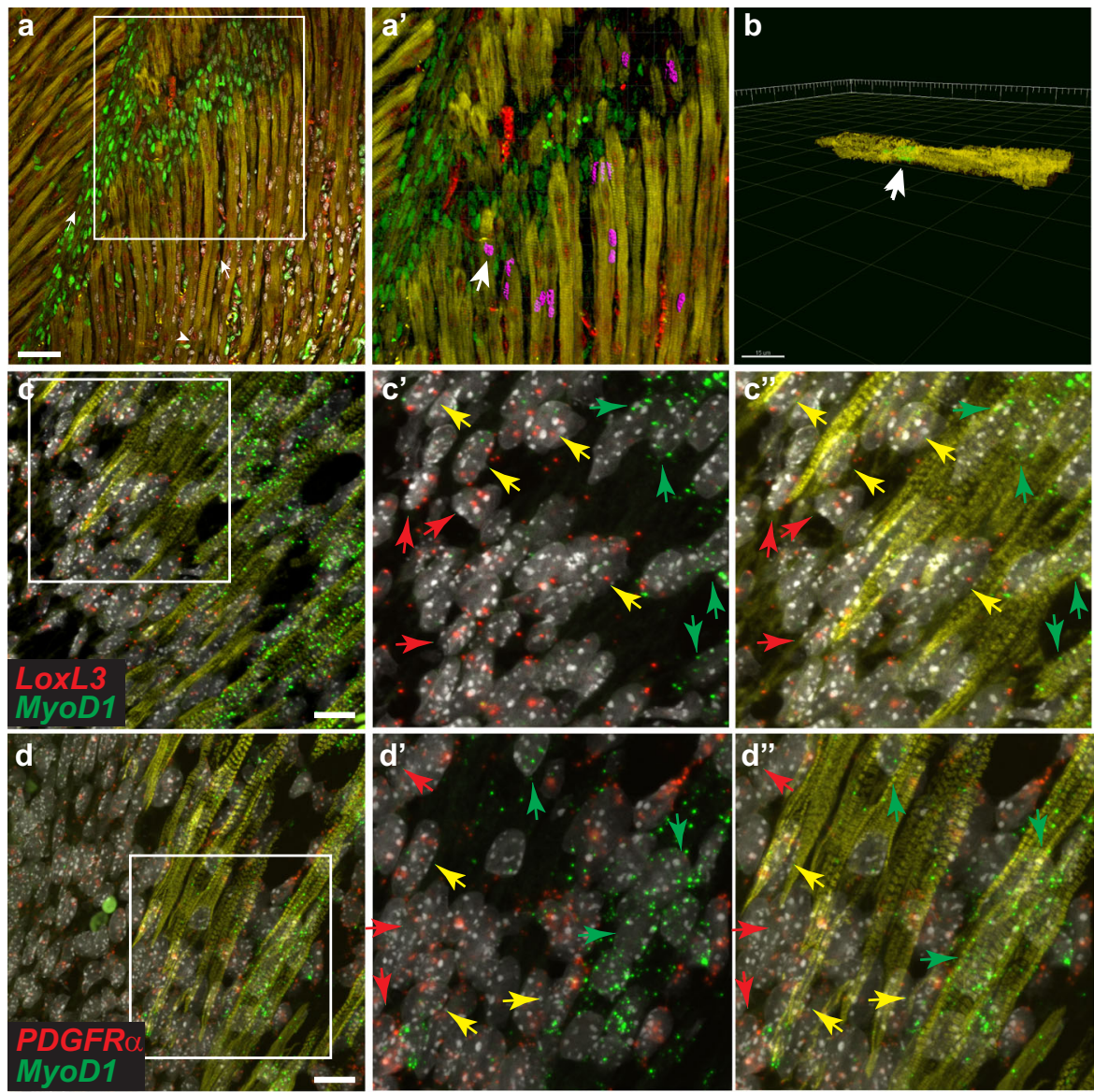

control

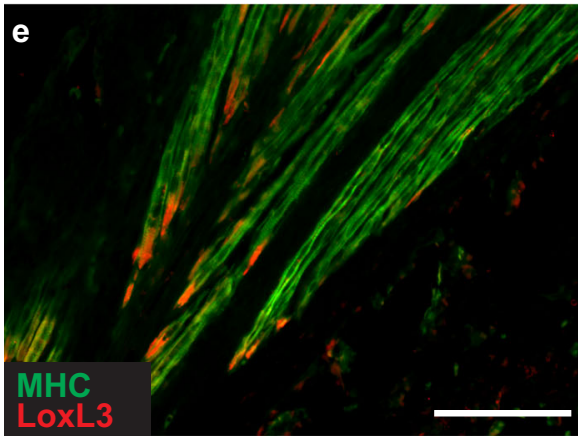

Prx1 $1^{\text {Cre }} ; \operatorname{Lox} L 3^{f / / \Delta}$

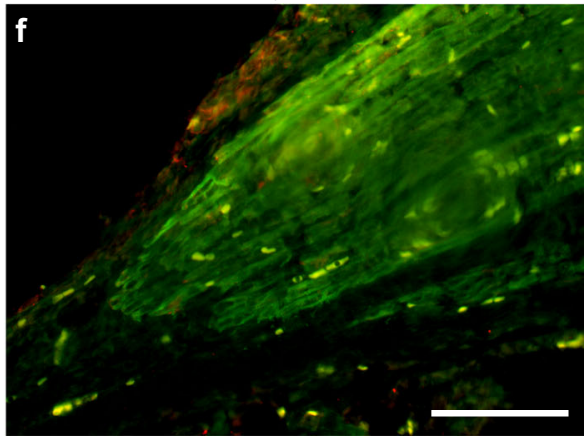

Fig. 5 LPM-derived cells fuse into myofibers along the MTJ. PO Prx 7 Cre; Rosat-ng limbs were subjected to immunostaining for MHC (yellow), EGFP (green) and tdTomato (red) (a) followed by IMARIS analysis demonstrates LPM-derived cells fuse into fibers at the proximity of the MTJ (a' purple; b lateral view of fiber section marked by white arrow in $\mathbf{a}^{\prime}$ ) (a, b). FISH for LoxL3 and MyoD1 (c-c') or PDGFR $\alpha$ and MyoD1 (d-d") on E15.5 limb sections. Tagged images show magnification of boxed areas in c, d. Staining demonstrates spatial localization of the distinct nuclei at the fiber tips those located towards the center of the muscle express only MyoD1 (green arrows in $\mathbf{c}^{\prime}, \mathbf{d}^{\prime}$ ), those closer to the tips (but also outside) express MyoD1 and fibroblastic markers (yellow arrows in $\mathbf{c}^{\prime}, \mathbf{d}^{\prime}$ ) and those outside of the fiber towards the tendon express only fibroblastic markers (red arrows in $\mathbf{c}^{\prime}, \mathbf{d}^{\prime}$ ). Immunostaining for MHC (green) and LoxL3 (red) on E17.5 control (e) and Prx 7 Cre LoxL $3^{f l / L}$ (f) demonstrates LoxL3 is missing at myofiber tips following its deletion in fibroblasts. IMARIS analyses (a-b), immunostaining and FISH results (c-f) are representative results derived from at least three independent embryos from distinct litters of the specific genotypes. Scale bar in $\mathbf{a}, \mathbf{e}, \mathbf{f}=50 \mu \mathrm{m}$, in $\mathbf{b}=15 \mu \mathrm{m}$ and in $\mathbf{c}, \mathbf{d}=10 \mu \mathrm{m}$.

The finding that LPM-derived fibroblasts fuse into the myofiber in proximity to the MTJs led us to test, whether LoxL3-expressing and PDGFR $\alpha$-expressing nuclei can be found in vivo within myofibers adjacent to the tips. FISH data for these fibroblast markers and for $M y o D 1$, establishes that adjacent to the myofiber tips, both within them but also outside, nuclei actively transcribing fibroblastic markers and $M y o D 1$ are present [Fig. $5 \mathrm{c}$,

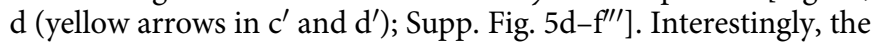

nuclei located towards the center of the fiber express only MyoD1 (green arrow in Fig. $5 c^{\prime}, d^{\prime}$ ) whereas those located farther away and outside of the fiber express only the fibroblastic marker (red arrow in Fig. $5 c^{\prime}, d^{\prime}$ ). Since some of the nuclei expressing both myogenic and fibrogenic markers are located at the tip of the fiber, where MHC expression is sometimes less prominent, we were not able to conclusively quantify the number of these nuclei that are fully contained within the fiber (Supp. Fig. 5g). 
In accordance with the above results, LoxL3 deletion in the LPM-derived cells using the $\operatorname{Pr} \times 1^{C r e}$ deleter line but not in the myogenic progenitors using the $\mathrm{Pax} 3^{\mathrm{Cre}}$ line (not shown), results in MTJ defects (Supp. Fig. 5h, i) and in loss of LoxL3 protein expression from the myofiber tips (Fig. 5e, f). Overall, these results demonstrate that the myofiber tips at the MTJ region encompass unique nuclei that are of LPM-origin that also express fibroblastic markers, and that this hybrid nature of the muscle fiber is critical for formation and maintenance of the MTJs.

\section{Discussion}

Here we explored the mechanisms underlying the building of the musculoskeletal system focusing on the MTJs. Single cell resolution techniques allowed us to revisit the paradigms depicting muscle development. We identified that LPM-derived fibroblasts transdifferentiate by switching on the myogenic program and fuse into the myofiber. We demonstrate this fusion is part of the normal developmental program generating syncytial myofibers with hybrid developmental origins. Our results suggest this process is essential for MTJ formation. Notably, in an accompanying paper D. Duprez and colleagues (Esteves de Lima, co-submitted) find this dual origin contribution to the myofibers at the MTJ occurs also in avian development. Reinforcing our observations, a unique group of nuclei expressing myogenic and fibrogenic genes (MTJ-B), the majority of which are shared by the dual identity cells, located at the MTJs, was recently identified in single nuclei sequencing of adult and regenerating myofibers ${ }^{32}$ further suggesting the hybrid nature of muscle fibers.

FISH analysis demonstrated LoxL3 is transcribed by fibroblasts. Accordingly, upon its deletion in the LPM-derived cells we observed LoxL3 protein loss. Interestingly, LoxL3 protein, and hence its loss, was observed in the myofibers. Notably, while myofiber nuclei are thought to be somite-derived, the rest of the limb's tissue, including the tendons and muscle connective tissues are derived from the LPM. Using live imaging and lineage tracing, we identified that LPM-derived fibroblasts can fuse into the elongating myofibers. Upon fusion, these fibroblasts maintain, at least partially, their original transcriptional program and thus facilitate the expression of fibroblast-specific genes in myofibers altogether leading to localized expression of fibroblast-specific genes in myofibers. We have previously demonstrated that LoxL3 oxidizes fibronectin along the myofiber tips. This oxidation promotes rapid activation of the integrin receptors expressed on the myofibers' termini ${ }^{12}$. Hence proper localization of LoxL3 enzymatic activity along the myofibers tips is critical for the fibers' adhesion along the forming MTJ. Thus, as a by-product of the LPM-derived cells' fusion to the myofiber tips, LoxL3 becomes localized to their termini and can then act locally along the MTJ.

Recent work has demonstrated the tendon-bone junctions is also composed of two cell types: tendon fibroblasts and chondrocytes ${ }^{33,34}$. Interestingly, this junction is dependent on TGF $\beta$ and BMP4 signaling 17,33,34. Notably, tendon development is highly dependent on TGF $\beta$ signaling ${ }^{35,36}$ and BMP4 activity is prominent in muscle fiber tips ${ }^{11}$. Our results also point to an involvement of these two pathways in the dual identity cells that contribute to the MTJs putting forward the possibility that cells with dual programs are required for generating junctions between tissues.

\footnotetext{
Methods

Mice. All experiments involving mice conform to the relevant regulatory standards (Technion IACUC and national animal welfare laws, guidelines and policies). All mice are housed in IVC's (Techniplast) according to space requirements defined by the NRC. All rooms are set to have $22^{\circ} \mathrm{C} \pm 2^{\circ}$ and humidity of $30-70 \%$. All HVAC parameters are controlled by a central computerized monitoring system. Light cycle is set to full light $10 \mathrm{~h}$ half-light $2 \mathrm{~h}$ and complete darkness $12 \mathrm{~h}$, light cycle is
} monitored by the computerized central system. Embryonic day (E) was staged according to Kaufmann ${ }^{37}$; noon of the day a vaginal plug was observed was marked as E0.5.

The LoxL3 allele and the $\operatorname{Prx} 1^{\text {Cre }}$ were previously described ${ }^{12,38}$, respectively. Rosa26R tdTomato (also known as Ai9) ${ }^{25}$ and Rosa $26^{n T n G}$ were purchased from JAX mice. All mice are kept and bred on a $\mathrm{C} 57 \mathrm{Bl} / 6$ background purchased from Envigo (https://www.envigo.com).

Mouse genotyping. Mice were PCR genotyped using the following primers:

LoxL3: LoxL3-5armWTF: GCCAGGGTGAAGTGAAAGAC; LoxL3-CritWTR: GATCTGGGATGCTGAAGACC; Tmla-5mut-R1: GAACTTCGGAATAGGAAC TTCG.

300 and $100 \mathrm{bp}$ represent wild-type and mutant PCR products, respectively. Cre: CreORF REV: ATCCAGGTTACGGATATAGT; CreORF FWD:

ATCCGAAAAGAAAACGTTGA. $500 \mathrm{bp}$ represent a cre positive PCR product. nTnG: nT-nG_1: CCAGGCGGGCCATTTACCGTAAG; nT-nG_2:

GGAGCGGGAGAAATGGATATG; nTnG 3: AAAGTCGCTCTGAGTTGTTAT. 603 and 320 bp represent wild-type and homozygous presence of nTnG PCR products, respectively.

tdTomato: RosaTomato WT_FWD: AAGGGAGCTGCAGTGGAGTA; RosaTomato WT_REV: CCGAAAATCTGTGGGAAGTC. Fragment size of $196 \mathrm{bp}$ represent the tomato transgene, while a fragment size of 297 bp represent the wildtype.

The complete set of primers used is shown in Supp. Table 1.

Fluorescent in situ hybridization (FISH). FISH was carried out using the RNAScope system according to the manufacturers' protocol ${ }^{39}$, on FFPE forelimb and hindlimb sections and also in primary cell cultures. Briefly, the sections were baked in a dry oven for $1 \mathrm{~h}$ at $60^{\circ} \mathrm{C}$, deparaffinized using xylene and ethanol, air dried and incubated in hydrogen peroxide for $10 \mathrm{~min}$. For the antigen retrieval step, slides were boiled in an anti-retrieval solution for $2 \mathrm{~min}$, transferred to ethanol for $3 \mathrm{~min}$ and air dried. Slides were then incubated with an RNAScope special protease for $30 \mathrm{~min}$. Designed target probes of LoxL3 (431359; RNAScope), MyoD1 (316081-C2; RNAScope), or PDGFR $\alpha$ (480661-C3; RNAScope) were added to the slides and incubated for $2 \mathrm{~h}$ at $40^{\circ} \mathrm{C}$. A series of probe amplifiers were added afterwards. At the end of the protocol, after adding the fluorophores, immunofluorescent staining was carried out. For cultured adherent cells, a similar protocol was carried out with the exception that the hydrogen peroxide incubation commenced directly after fixation (4\% PFA).

All assays were repeated at least at three time points using different cell cultures or tissue sections from distinct embryos/mice.

Immunohistochemistry. Section and whole-mount immunohistochemistry were performed essentially as previously described ${ }^{40}$. Briefly, $5 \mu \mathrm{m}$ paraffin or $10 \mu \mathrm{m}$ OCT embedded tissue sections were incubated over-night at $4{ }^{\circ} \mathrm{C}$ with the following antibodies at the concentrations below. Antigen retrieval was performed only when LoxL3 antibody was used. Wholemount immunostaining was carried out essentially with the same primary antibody concentrations although incubation periods were extended to 2 weeks in $4 \mathrm{C}$ (for both primary and secondary antibodies). The following antibodies were used: anti-Myosin (A4.1025, 1:300; DSHB); anti-GFP (A6455, 1:500; Invitrogen); anti-RFP (5F8, 1:500; Chromotek); antiLOXL3 $^{12}(1: 100)$

All assays were repeated at least at three time points using different cell cultures or tissue sections from distinct embryos/mice.

Fluorescent cell sorting. $\operatorname{Pr} x 1^{\text {Cre }} ; \operatorname{Rosa} 26^{n T n G} \mathrm{P} 0$ neonate limb muscles were sliced into pieces, incubated with collagenase for $1 \mathrm{~h}$ and then with trypsin for half an hour. Tissues were then centrifuged for $15 \mathrm{~min}$ at $317 \times g$, resuspended in DMEM (containing 1\% L-glutamine and $1 \%$ penicillin and streptomycin (PS) $+10 \%$ FCS, filtered, re-centrifuged, re-suspended in BIO-AMF-2 (Biological Industries) and plated on gelatin coated plates. Cells were then cultured for $24 \mathrm{~h}$ and then sorted using FACSAria ${ }^{\mathrm{m}}$ IIIu (BD Biocsiences) and data analyzed using BD FACS Diva8.0.1 software. Gating strategy was the following: Cells were initially gated on FSC-A/SSC-A to exclude outliers and cell debris. Gated cells were then single gated twice based on FSC-W/FSC-H and then by SSC-W/SSC-H. Single cells were then plotted on B530/30 (GFP) versus YG610/20 (RFP). Single RFP expressing cells and single GFP expressing cell were sorted and cultured.

Generation of primary cultures. P0 neonate limb muscles were sliced into pieces, incubated with collagenase for $1 \mathrm{~h}$ and then with trypsin for half an hour. Tissues were then centrifuged for $15 \mathrm{~min}$ at $317 \times g$, resuspended in DMEM (containing $1 \%$ L-glutamine and $1 \%$ penicillin and streptomycin (PS) $+10 \%$ FCS, filtered, recentrifuged, re-suspended in BIO-AMF-2 (Biological Industries) and plated on gelatin coated plates. Differentiation was induced by incubating the cells in DMEM (containing 1\% L-glutamine and 1\% PS) $+4 \%$ horse serum for 3 days.

10x Genomics single cell general cell preparation. Single cell separation and library construction according to $10 \times$ protocol (Chromium Single Cell $3^{\prime}$ Library \& Gel Bead Kit v2). Briefly, the MTJ region was dissected from P0 neonates and single 
cells were isolated (as described) and suspended with BIO-AMF-2 (Biological Industries). The cells were mixed thoroughly using a wide-bore pipette tip and counted. Cells were centrifuged at $317 \times g$ for $5 \mathrm{~min}$ at RT, suspended with $0.04 \%$ BSA PBS, re-centrifuged and then re-suspended with the appropriate volume of $0.04 \%$ BSA PBS to achieve a target cell concentration in the range of 700-1200 cell/ $\mu \mathrm{l}$. Following cell isolation, library construction was immediately carried out. Cell Ranger software (V3.0.2) was used for data QC and extraction of transcripts' counts from raw data.

Bioinformatic analysis. Seurat R package (Seurat_3.0.0.9100) was used for filtering, clustering and expression distribution of selected cluster-specific genes. SingleR R Package (SingleR_0.2.2) was used for unbiased cell type recognition of scRNAseq. Cells with the following parameters were excluded: $>8 \%$ mitochondrial UMI counts; less than 200 unique gene counts; over 4000 unique gene counts. Overall, 10,456 cells entered the analysis and 9238 cells were used for the bioinformatics analysis after filtration. In addition, genes detected in less than three cells were filtered out. WebGestalt (WEB-based Gene SeT AnaLysis Toolkit) a functional enrichment analysis web tool and Ingenuity Pathway Analysis (Qiagen) were used to identify specific pathways and processes within the data.

RNA velocity analysis was conducted as follows: A loom file from $10 \times$ output using velocyto.R with default parameters was used ${ }^{19}$. In summary estimating RNA Velocity using Seurat was created and imported to R and an RNA velocity analysis was conducted for the whole data. The cluster labels from the initial analysis were assigned to the velocity analysis in order to compare between the results. Cells that didn't assign to any initial cluster were assigned as cluster " 0 ". RNA velocity was calculated on this data. Furthermore, cells that were labeled as fibroblasts, myoblasts, satellite cells, myocytes, and dual identity were extracted and analyzed using RNA velocity without re-clustering the data.

Reporting summary. Further information on research design is available in the Nature Research Reporting Summary linked to this article.

\section{Data availability}

Source data are provided with this paper. The datasets generated and/or analyzed during the current study are available in the GEO repository [https://www.ncbi.nlm.nih.gov/geo/ query/acc.cgi? acc $=$ GSE168153].

Received: 6 July 2020; Accepted: 3 June 2021;

Published online: 22 June 2021

\section{References}

1. Kieny, M. \& Chevallier, A. Autonomy of tendon development in the embryonic chick wing. J. Embryol. Exp. Morphol. 49, 153-165 (1979).

2. Schweitzer, R. et al. Analysis of the tendon cell fate using Scleraxis, a specific marker for tendons and ligaments. Development 128, 3855-3866 (2001).

3. Tajbakhsh, S. \& Buckingham, M. The birth of muscle progenitor cells in the mouse: spatiotemporal considerations. Curr. Top. Dev. Biol. 48, 225-268 (2000).

4. Wachtler, F., Christ, B. \& Jacob, H. J. On the determination of mesodermal tissues in the avian embryonic wing bud. Anat. Embryol. 161, 283-289 (1981).

5. Gu, J. M. et al. An NF-kappaB-EphrinA5-dependent communication between $\mathrm{NG} 2(+)$ interstitial cells and myoblasts promotes muscle growth in neonates. Dev. Cell 36, 215-224 (2016)

6. Kitiyakara, A. \& Angevine, D. M. A study of the pattern of postembryonic growth of M. Gracilis in mice. Dev. Biol. 8, 322-340 (1963).

7. Williams, P. E. \& Goldspink, G. Longitudinal growth of striated muscle fibres. J. Cell Sci. 9, 751-767 (1971)

8. Tsujimura, T., Kinoshita, M. \& Abe, M. Response of rabbit skeletal muscle to tibial lengthening. J. Orthop. Sci.11, 185-190 (2006).

9. Zhang, M. \& McLennan, I. S. During secondary myotube formation, primary myotubes preferentially absorb new nuclei at their ends. Dev. Dyn. 204, 168-177 (1995).

10. Edom-Vovard, F., Bonnin, M. A. \& Duprez, D. Misexpression of Fgf-4 in the chick limb inhibits myogenesis by down-regulating Frek expression. Dev. Biol. 233, 56-71 (2001)

11. Wang, H. et al. Bmp signaling at the tips of skeletal muscles regulates the number of fetal muscle progenitors and satellite cells during development. Dev. Cell 18, 643-654 (2010).

12. Kraft-Sheleg, O. et al. Localized LoxL3-dependent fibronectin oxidation regulates myofiber stretch and integrin-mediated adhesion. Dev. Cell 36, 550-561 (2016).

13. Baumeister, A., Arber, S. \& Caroni, P. Accumulation of muscle ankyrin repeat protein transcript reveals local activation of primary myotube endcompartments during muscle morphogenesis. J. Cell Biol. 139, 1231-1242 (1997).
14. Esteves de Lima, J., Bonnin, M. A., Birchmeier, C. \& Duprez, D. Muscle contraction is required to maintain the pool of muscle progenitors via YAP and NOTCH during fetal myogenesis. eLife https://doi.org/10.7554/ eLife.15593 (2016).

15. Jo, C. H., Lim, H. J. \& Yoon, K. S. Characterization of tendon-specific markers in various human tissues, tenocytes and mesenchymal stem cells. Tissue Eng. Regener. Med. 16, 151-159 (2019).

16. Subramanian, A. \& Schilling, T. F. Thrombospondin- 4 controls matrix assembly during development and repair of myotendinous junctions. eLife https://doi.org/10.7554/eLife.02372 (2014).

17. Maeda, T. et al. Conversion of mechanical force into TGF-beta-mediated biochemical signals. Curr. Biol. 21, 933-941 (2011).

18. Wang, J., Duncan, D., Shi, Z. \& Zhang, B. WEB-based GEne SeT AnaLysis Toolkit (WebGestalt): update 2013. Nucleic Acids Res. 41, W77-W83 (2013)

19. La Manno, G. et al. RNA velocity of single cells. Nature 560, 494-498 (2018)

20. Logan, M. et al. Expression of Cre Recombinase in the developing mouse limb bud driven by a Prxl enhancer. Genesis 33, 77-80 (2002).

21. Martin, J. F. \& Olson, E. N. Identification of a prx1 limb enhancer. Genesis 26, 225-229 (2000)

22. Colasanto, M. P. et al. Development of a subset of forelimb muscles and their attachment sites requires the ulnar-mammary syndrome gene Tbx3. Dis. Model Mech. 9, 1257-1269 (2016)

23. Seo, H. S. \& Serra, R. Deletion of Tgfbr2 in Prx1-cre expressing mesenchyme results in defects in development of the long bones and joints. Dev. Biol. 310, 304-316 (2007).

24. Prigge, J. R. et al. Nuclear double-fluorescent reporter for in vivo and ex vivo analyses of biological transitions in mouse nuclei. Mamm. Genome https://doi. org/10.1007/s00335-013-9469-8 (2013).

25. Madisen, L. et al. A robust and high-throughput Cre reporting and characterization system for the whole mouse brain. Nat. Neurosci. 13, 133-140 (2010).

26. Sampath, S. C., Sampath, S. C. \& Millay, D. P. Myoblast fusion confusion: the resolution begins. Skelet. Muscle 8, 3 (2018).

27. van Niel, G., D'Angelo, G. \& Raposo, G. Shedding light on the cell biology of extracellular vesicles. Nat. Rev. Mol. Cell Biol. 19, 213-228 (2018).

28. Yamashita, Y. M., Inaba, M. \& Buszczak, M. Specialized intercellular communications via cytonemes and nanotubes. Annu. Rev. Cell Dev. Biol. 34, 59-84 (2018)

29. Millay, D. P. et al. Myomaker is a membrane activator of myoblast fusion and muscle formation. Nature 499, 301-305 (2013).

30. Goh, Q. \& Millay, D. P. Requirement of myomaker-mediated stem cell fusion for skeletal muscle hypertrophy. eLife https://doi.org/10.7554/eLife.20007 (2017).

31. Petrany, M. J., Song, T., Sadayappan, S. \& Millay, D. P. Myocyte-derived myomaker expression is required for regenerative fusion but exacerbates membrane instability in dystrophic myofibers. JCI Insight https://doi.org/ 10.1172/jci.insight.136095 (2020).

32. Kim, M. et al. Single-nucleus transcriptomics reveals functional compartmentalization in syncytial skeletal muscle cells. Nat. Commun. 11, 6375 (2020)

33. Blitz, E., Sharir, A., Akiyama, H. \& Zelzer, E. Tendon-bone attachment unit is formed modularly by a distinct pool of Scx- and Sox9-positive progenitors. Development 140, 2680-2690 (2013).

34. Zelzer, E., Blitz, E., Killian, M. L. \& Thomopoulos, S. Tendon-to-bone attachment: from development to maturity. Birth defects research. Part C. Embryo Today 102, 101-112 (2014).

35. Havis, E. et al. Transcriptomic analysis of mouse limb tendon cells during development. Development141, 3683-3696 (2014).

36. Pryce, B. A. et al. Recruitment and maintenance of tendon progenitors by TGFbeta signaling are essential for tendon formation. Development 136, 1351-1361 (2009)

37. Kaufmann, M. H. The Atlas of Mouse Development. (Elsevier, 1992).

38. Logan, M. \& Tabin, C. Targeted gene misexpression in chick limb buds using avian replication-competent retroviruses. Methods 14, 407-420 (1998).

39. Wang, F. et al. RNAscope: a novel in situ RNA analysis platform for formalinfixed, paraffin-embedded tissues. J. Mol. Diagn. 14, 22-29 (2012).

40. Hasson, P. et al. Tbx 4 and Tbx 5 acting in connective tissue are required for limb muscle and tendon patterning. Dev. Cell 18, 148-156 (2010).

\section{Acknowledgements}

We are grateful to Dr. E. Suss-Toby and M. Holdengreber from the BCF Bioimaging Center (Faculty of Medicine, Technion); to A. Shemesh for 3D-image analysis; to L. Linde and R. Modai-Hod from the BCF Genomics Center (Faculty of Medicine, Technion) for bioinformatic analyses to members of the animal facility for excellent technical assistance. We further thank T. Schultheiss for critical reading of the manuscript and for fruitful discussions. P.H. was supported by grants from the Israeli Science Foundation $(1072 / 13)$ and $(1111 / 18)$, by A.D.I. (Association Duchenne Israel) and by the Rappaport 
Family Institute. The work in the laboratory of D.P.M. is supported by grants from the National Institutes of Health (R01AR068286, R01AG059605) and Pew Charitable Trusts.

\section{Author contributions}

W.Y. conducted the majority of the experiments. O.K.S participated in analyzing the LoxL3 MTJ phenotypes. S.Z.E. and S.M. assisted in cell culture assays. C.S. and D.P.M. participated in the myomaker experiments. W.Y. and P.H planned the study, supervised and analyzed the data. D.P.M. and P.H. wrote the manuscript.

\section{Competing interests}

The authors declare no competing interests.

\section{Additional information}

Supplementary information The online version contains supplementary material available at https://doi.org/10.1038/s41467-021-24159-9.

Correspondence and requests for materials should be addressed to P.H.

Peer review information Nature Communications thanks Noriaki Ono and the other anonymous, reviewer(s) for their contribution to the peer review of this work.
Reprints and permission information is available at http://www.nature.com/reprints

Publisher's note Springer Nature remains neutral with regard to jurisdictional claims in published maps and institutional affiliations.

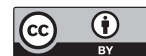

Open Access This article is licensed under a Creative Commons Attribution 4.0 International License, which permits use, sharing, adaptation, distribution and reproduction in any medium or format, as long as you give appropriate credit to the original author(s) and the source, provide a link to the Creative Commons license, and indicate if changes were made. The images or other third party material in this article are included in the article's Creative Commons license, unless indicated otherwise in a credit line to the material. If material is not included in the article's Creative Commons license and your intended use is not permitted by statutory regulation or exceeds the permitted use, you will need to obtain permission directly from the copyright holder. To view a copy of this license, visit http://creativecommons.org/ licenses/by/4.0/.

(C) The Author(s) 2021 